\title{
Scope definition and resection significance of No. 12a group lymph nodes in gastric cancer
}

\author{
YONGJIAN HUANG ${ }^{1,2^{*}}$, GUANGWEI ZHU ${ }^{1,2^{*}}$, WEI ZHENG ${ }^{1,2}$, JIN HUA $^{1,2}$, \\ SHUGANG YANG ${ }^{1,2}$, JINFU ZHUANG ${ }^{1,2}$, JINZHOU WANG $^{1,2}$ and JIANXIN YE ${ }^{1,2}$ \\ ${ }^{1}$ Department of Gastrointestinal Surgery 2 Section, The First Hospital Affiliated to Fujian Medical University; \\ ${ }^{2}$ Key Laboratory of Ministry of Education for Gastrointestinal Cancer, Fujian \\ Medical University, Fuzhou, Fujian 350004, P.R. China
}

Received January 20, 2016; Accepted April 20, 2016

DOI: $10.3892 / \operatorname{mco} .2016 .911$

\begin{abstract}
A discrepancy exists between the 7th edition guidelines of the American Joint Committee on Cancer (AJCC) and the 3rd edition Japanese treatment guidelines in terms of the classification of No. 12a lymph nodes as regional or distant lymph nodes in D2 lymphadenectomy for gastric cancer. The scope definition of No. 12a lymph nodes has yet to be fully elucidated. The present study aimed to assess the appropriateness of reclassifying No. 12a lymph node metastasis as distant metastasis according to the survival rate outcome, and to provide a clear and practical definition of the No. 12a group lymph nodes of gastric cancer. A retrospective analysis was performed on patients with gastric cancer who underwent standard or greater lymphadenectomy between January 2000 and December 2009 to find an association between No. 12a node metastasis and survival outcome. The present study first presented a clear and practical scope definition of the No. 12a group lymph nodes of gastric cancer, according to our clinical experiences and practices (Table I and Fig. 1). The survival outcome of patients with gastric cancer and No. 12a lymph node metastasis was poorer compared with that of patients with no No. 12a lymph node metastasis $(\mathrm{P}=0.0003)$. The results were similar in stage III patients with gastric cancer $(\mathrm{P}<0.0001)$. However, the survival outcome of patients was similar with or without No. 12a lymph node metastasis in stage IV gastric cancer $(\mathrm{P}=0.1968)$. Cox regression analysis revealed that the AJCC stage was independently associated with an unfavorable cumulative survival rate. Logistic regression analysis
\end{abstract}

Correspondence to: Professor Jianxin Ye, Department of Gastrointestinal Surgery 2 Section, The First Hospital Affiliated to Fujian Medical University, 20 Cha-Zhong Road, Fuzhou, Fujian 350004, P.R. China

E-mail: yejianxinfuyi@126.com

${ }^{*}$ Contributed equally

Key words: gastric cancer, No. 12a lymph nodes, metastasis, lymphadenectomy revealed that tumor location, AJCC stage, intravascular cancer emboli and nerve invasion were associated with No. 12a lymph node metastasis. In conclusion, the data in the present study suggested that No. 12a lymph node metastasis is associated with distant metastasis, and therefore they concur with the 7th edition AJCC gastric cancer guidelines, which appear to be correct in terms of considering No. 12a lymph node metastasis as distant metastasis.

\section{Introduction}

Gastric cancer is a relatively common type of cancer, and is the second leading cause of cancer mortality in the world. In excess of 700,000 deaths of patients with gastric cancer, and almost 1,000,000 new gastric cancer cases, occur globally each year (1). Gastric cancer has an extremely poor prognosis. The 5 -year survival rates for gastric cancer are low in most countries, at $<30 \%$ (2). Surgical resection is the only potentially curative therapy for gastric cancer. However, only a minority of patients with gastric cancer are suitable for surgical treatment, predominantly due to the high proportion of advanced tumors at the time of presentation (2). Thus, radical operation remains the most important therapeutic means for patients with gastric cancer to achieve long-term survival. The classification of the lymphadenectomy of gastric cancer is a vital link for improving treatment. The latest lymph node $(\mathrm{N})$ staging of gastric cancer is determined by calculating the number of lymph nodes $(3,4)$, which requires lymph node harvesting to accurately determine the staging for patients with gastric cancer. Thus, the anatomical structure of complex tissues of lymph node resection, such as No. 12a lymph nodes, is receiving increased attention.

The 7th edition guidelines of the American Joint Committee on Cancer (AJCC) for gastric cancer have been rigorously debated $(5,6)$. One of the changes in these guidelines is that No. 12a lymph nodes are no longer assigned to D2 lymphadenectomy [numerous surgeons agree that D2 lymphadenectomy is a standard surgical procedure for gastric cancer $(7,8)]$, and No. 12a metastasis has been reclassified accordingly as distant metastasis.

However, the 3rd edition of the Japanese treatment guidelines and the 6th edition of the AJCC guidelines for gastric 
Table I. Scope definition of No. 12a lymph nodes according to the AJCC/UICC and Japanese treatment guidelines, and our scope definition.

AJCC/UICC guidelines and the Japanese

treatment guidelines

Our scope definition

Lymph nodes along the proper hepatic artery, in the caudal half between the confluence of the right and left hepatic ducts and the upper border of the pancreas.
1: Upper border: The confluence of the right and left hepatic artery.

2: Lower border: The upper border of the pancreas of the origin of the proper hepatic artery.

3: Offside border: The left of the bile duct.

4: Left side border: The left side border of the peritoneum fusion place of ligamentum hepatoduodenale, including lymphoid tissue.

5: Front border: The anterior hepatoduodenal ligament.

6: Posterior border: The front of the portal vein (Fig. 1).

AJCC, American Joint Committee on Cancer; UICC, Union for International Cancer Control.

cancer concur that No. 12a lymph node metastasis is a type of regional metastasis from a primary gastric cancer, and should be dissected during D2 lymphadenectomy to improve patient outcome $(9,10)$. The difference between the guidelines may cause confusion in surgeons.

The scope definition of No. 12a group lymph nodes is undefinable according to the AJCC guidelines, Japanese treatment guidelines and the National Comprehensive Cancer Network. None of these guidelines are able to describe the scope definition of No. 12a group lymph nodes for gastric cancer. These guidelines completely reference the guidelines for biliary carcinoma processing, which indicate that No. 12a group lymph nodes may be located along the proper hepatic artery (11).

The aim of the present study was to provide a clear and practical scope definition of No. 12a group lymph nodes according to our clinical experiences and practices, and to evaluate the clinical importance and survival outcomes of patients with gastric cancer with No. 12a lymph node metastasis following D2 lymphadenectomy.

\section{Materials and methods}

The present study was approved by the Institutional Review Board of the first Hospital Affiliated to Fujian Medical University, and informed consent was obtained according to institutional regulations. Written informed consent for further clinical research was obtained from participants for their clinical records.

Clinical data collection. Data obtained from patients with gastric cancer who received gastrectomy plus D2 or greater lymphadenectomy between January 2000 and December 2009 at the first Hospital Affiliated to Fujian Medical University were retrospectively analyzed. The inclusion criteria were as follows: i) advanced gastric cancer; ii) carcinoma (including adenocarcinoma, mucinous or signet ring adenocarcinoma) confirmed by histopathology; iii) D2 or greater lymphadenectomy; iv) patients did not receive neoadjuvant chemotherapy or chemoradiotherapy prior to surgical operation.

Follow-up was conducted every three to six months for the first three years, and once a year thereafter. All patients were

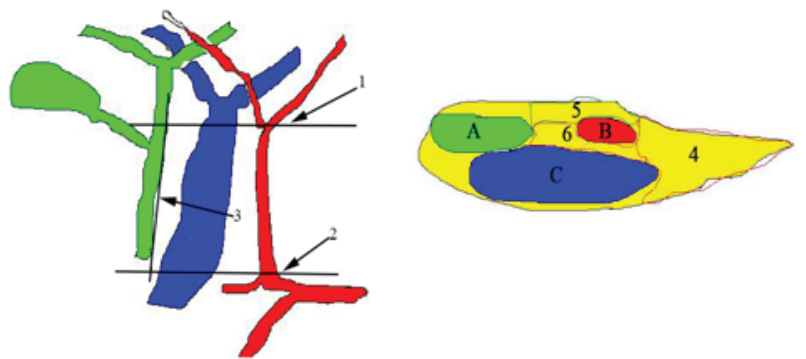

Figure 1. The level and cross section diagram of the No. 12a group lymph nodes. (A) Choledoch (shown in green); (B) hepatic artery (shown in red); (C) portal vein (shown in blue).

followed up by out-patient review and telephone interviews. The clinicopathological and follow-up findings were collected and recorded in the database.

Surgery. All patients in the study underwent total or distal gastrectomy, depending on the location and macroscopic appearance of the tumor. Distal and total gastrectomies were performed principally for tumors located in the lower third, middle, or upper third of the stomach, and for tumors occupying the entire stomach. The strategy for lymph node dissections was determined by using a standardized technique according to the guidelines of the 2010 Japanese Classification of Gastric Cancer and Gastric Cancer Treatment Guidelines, edited by the Japanese Gastric Cancer Association (9), which consider No. 12a lymph node metastasis as regional progression.

In the present study, a clear and practical scope definition of the No. 12a group lymph nodes of gastric cancer is provided according to our clinical experiences and practices. Given that the scope definition of No. 12a group lymph nodes is undefinable according to the guidelines of AJCC/Union for International Cancer Control and Japanese treatment guidelines, none of the guidelines are able to describe the scope definition of No. 12a group lymph nodes for gastric cancer (Table I and Fig. 1).

The surgical procedures of No. 12a node resection were as follows. First, the ligamentum hepatoduodenale was exposed, the gastroduodenum segment was stretched, and the ligamentum hepatoduodenale was flattened. The anterior 
Table II. Comparison of clinical parameters in patients with gastric cancer with or without No. 12a group lymph node metastasis.

\begin{tabular}{|c|c|c|c|c|}
\hline Clinicopathological parameters & No. $12 \mathrm{a}(-)^{\mathrm{a}}$ & No. $12 \mathrm{a}(+)^{\mathrm{b}}$ & $\mathrm{N}$ & P-value \\
\hline Age (year) & & & & 0.5422 \\
\hline$<61$ & 43 & 13 & 56 & \\
\hline$\geq 61$ & 92 & 21 & 113 & \\
\hline Gender & & & & 0.2927 \\
\hline Male & 92 & 27 & 119 & \\
\hline Female & 43 & 7 & 50 & \\
\hline Histological grade & & & & $0.0006^{\mathrm{e}}$ \\
\hline $\mathrm{H}+\mathrm{M}^{\mathrm{c}}$ & 58 & 4 & 62 & \\
\hline $\mathrm{L}+\mathrm{O}^{\mathrm{d}}$ & 77 & 30 & 107 & \\
\hline Tumor location & & & & 0.5689 \\
\hline Upper & 53 & 12 & 65 & \\
\hline Middle & 22 & 2 & 24 & \\
\hline Lower & 60 & 20 & 80 & \\
\hline AJCC stage & & & & $0.0063^{\mathrm{e}}$ \\
\hline $\mathrm{I}+\mathrm{II}$ & 30 & 1 & 31 & \\
\hline III+IV & 105 & 33 & 138 & \\
\hline T stage & & & & $0.0002^{\mathrm{e}}$ \\
\hline $\mathrm{T} 1-\mathrm{T} 2$ & 34 & 0 & 34 & \\
\hline $\mathrm{T} 3-\mathrm{T} 4$ & 101 & 34 & 135 & \\
\hline Lymph node metastasis & & & & $0.0008^{\mathrm{e}}$ \\
\hline No & 30 & 0 & 30 & \\
\hline Yes & 105 & 34 & 139 & \\
\hline Intravascular cancer emboli & & & & $0.0001^{\mathrm{e}}$ \\
\hline No & 67 & 4 & 71 & \\
\hline Yes & 68 & 30 & 98 & \\
\hline Nerve invasion & & & & $0.0001^{\mathrm{e}}$ \\
\hline No & 84 & 6 & 90 & \\
\hline Yes & 51 & 28 & 79 & \\
\hline Tumor size & & & & $0.0070^{\mathrm{e}}$ \\
\hline$<5 \mathrm{~cm}$ & 66 & 8 & 74 & \\
\hline$\geq 5 \mathrm{~cm}$ & 69 & 27 & 96 & \\
\hline
\end{tabular}

The 7th edition of the American Joint Committee on Cancer (AJCC) staging system was used. ${ }^{\text {ano. }}$ 12a lymph nodes negative group; ${ }^{b}$ No. 12a lymph nodes positive group; chigh differentiation and moderate differentiation; ${ }^{d}$ poor and other types of differentiation, including

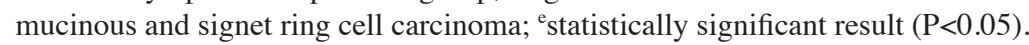

hepatoduodenal ligament was opened to the confluence of the right and left hepatic arteries. Secondly, the perivascular sheath along the hepatic artery was opened to the upper border of the pancreas at the origin of the proper hepatic artery, including the left side border peritoneum fusion site of the ligamentum hepatoduodenale, also including the lymphoid tissue. Finally, the perivascular sheath of the portal vein was opened, and the lymphoid tissue at the front of portal vein was cleared. An electrotome (Force FXTM-8C; Tyco Healthcare Group LP, Boulder, CO, USA) was used in the whole resection process for sharp dissection.

Statistical analysis. All statistical analyses were performed using IBM SPSS software (v. 19.0; IBM, Armonk, NY, USA). The categorical variables were compared by using the Chi-squared test or Fisher's test. Survival curves were calculated using the Kaplan-Meier method, and compared using the log-rank test. Logistic regression analysis was used to assess the risk factors of No. 12a lymph node metastasis. A Cox proportional hazard model was used to explore the independent factors of survival status on the basis of the variables selected in univariate analysis. $\mathrm{P}<0.05$ was considered to indicate a statistically significant value for each analysis.

\section{Results}

Clinicopathological characteristics. Among the 169 patients who underwent No. 12a lymph node resection, $119(70 \%)$ patients were men and $50(40 \%)$ were women. The mean age at diagnosis was $64.1 \pm 12$ years, and follow-up periods 
were between 0.9 and 77.5 months (median, 40 months). A total number of 34 (20\%) patients had an involvement of No. 12a lymph nodes [No. 12a $\left.\left(^{+}\right)\right]$. The mean positive yield was 2.4 (range, 1-7) No. 12a lymph nodes. The data associated with the clinical and pathological characteristics are shown in Table II. No differences were exhibited in age, gender or tumor location between the No. $12 \mathrm{a}(+)$ patients and the No. $12 \mathrm{a}(-)$ patients. No. $12 \mathrm{a}(+)$ patients did have significantly higher clinicopathological parameters $(\mathrm{P}<0.05)$, including $\mathrm{T}$ stage, and exhibited severe histological grade, a number of metastatic lymph nodes, numerous, commonly intravascular, cancer emboli, severe nerve invasion and large tumor size. All surgical procedures were performed by the identical surgical team.

Significance of the survival outcome of patients with No. 12 a lymph nodes. A comparison between patients with or without No. 12a lymph node metastasis revealed that those with No. 12a lymph node metastasis had a markedly poorer survival outcome (Fig. 2). A similar result was found between patients with or without No. 12a lymph node metastasis in stage III patients (Fig. 3). However, in the stage IV patients, no differences were observed in the survival outcome between the No. $12 \mathrm{a}(+)$ group and the No. 12a(-) group (Fig. 4). Univariate analysis was performed for clinicopathological data that possibly affected survival outcomes. Multivariate analysis was performed using the variables that were significant in the univariate analysis. Cox regression analysis revealed that the AJCC stage was the only prognostic factor that was independently associated with an unfavorable cumulative survival rate (Table III).

Influence factors of No. 12 a lymph node metastasis. Logistic regression analysis revealed that tumor location, the AJCC stage, intravascular cancer emboli and nerve invasion were associated with No. 12a lymph node metastasis (Table IV).

\section{Discussion}

Gastric cancer remains one of the most common causes of cancer-associated mortality in the world. Surgical resection is a curative treatment that is available for advanced cases, and lymphadenectomy is an important part of curative resection (12). Lymph node radical dissections for advanced gastric cancer are theoretically able to increase the patient survival rate. Thus, D2 dissection is a standard procedure for patients with gastric cancer. Since the 1990s, this procedure has been increasingly employed by surgeons to treat gastric cancer $(7,8,13)$.

Two major staging systems are used for gastric cancer: The Japanese treatment guidelines, and the AJCC guidelines. The majority of East Asian countries use both guidelines, although the AJCC guidelines are used worldwide. Both the 6th and 7th editions of the AJCC guidelines for gastric cancer base D2 lymphadenectomy on the tumor position.

However, a number of novel and carefully considered changes are present in the 7th edition of the AJCC guidelines for gastric cancer, for example, the exclusion of No. 12a lymph node dissection from D2 lymphadenectomy. However, these changes were not explained properly. This issue provided the impetus to conduct the present study.

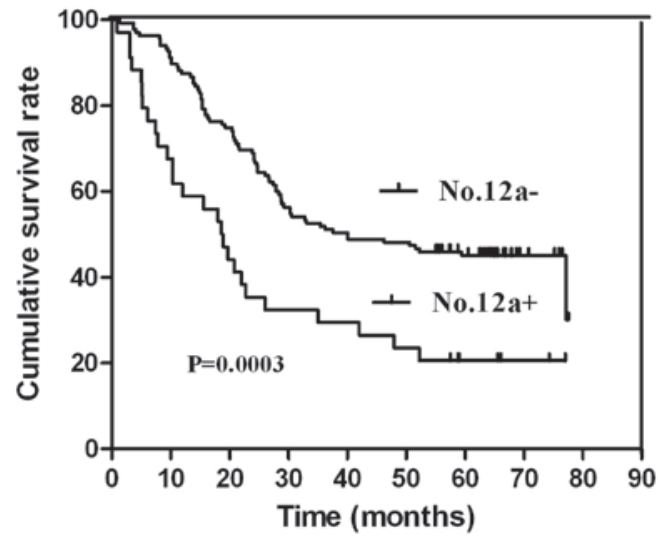

Figure 2. Survival curves for patients with gastric cancer according to No. 12a group lymph nodes (negative or positive; No.12a+ and No.12a-, respectively.). The survival rate of the No. 12a lymph nodes positive group was lower than that of the No. 12a lymph nodes negative group $(\mathrm{P}=0.003)$.

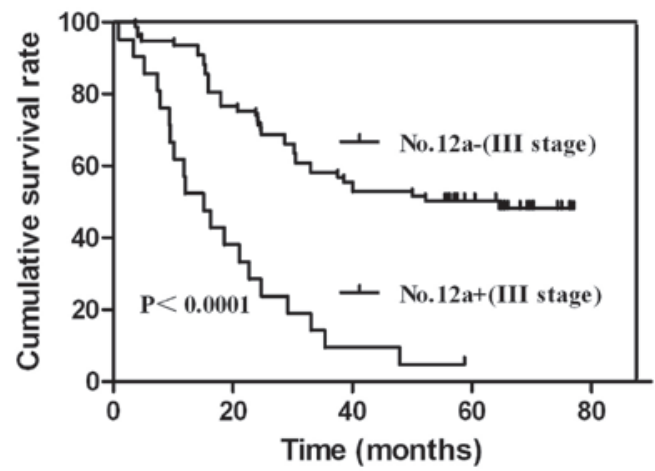

Figure 3. Survival curves according to No. 12a lymph nodes negative or positive groups in stage III patients with gastric cancer [No.12a-(III stage) and No.12a+(III stage), respectively]. The survival rate of the No. 12a lymph nodes positive group was lower than the survival rate of No. 12a lymph nodes negative group in the stage III patients with gastric cancer $(\mathrm{P}<0.0001)$.

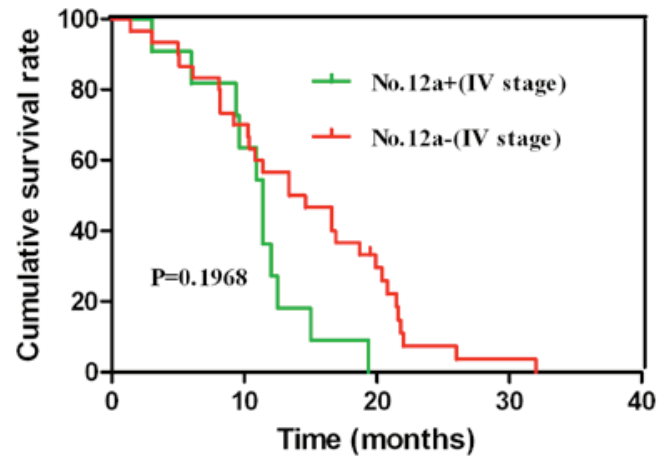

Figure 4.Comparison of the survival rate of stage IV patients with No.12alymph node metastasis, and those with other distant metastases [No.12a+(IV stage) and No.12a-(IV stage), respectively]. In the stage IV patients, no significant differences were observed for the survival outcome between the No. 12a positive and negative groups $(\mathrm{P}=0.1968)$.

On critically assessing the available information, no clear definition was identified to exist for No. 12a lymph nodes. Therefore, in the present study, a clear and practical definition of No. 12a group lymph nodes has been provided according 
Table III. Univariate and multivariate analysis of prognostic factors for the cumulative survival rate.

\begin{tabular}{|c|c|c|c|c|c|c|}
\hline \multirow[b]{2}{*}{ Factor } & \multicolumn{3}{|c|}{ Univariate analysis $^{\mathrm{a}}$} & \multicolumn{3}{|c|}{ Multivariate analysis } \\
\hline & OR & $95 \% \mathrm{CI}$ & P-value & OR & $95 \% \mathrm{CI}$ & P-value \\
\hline Age & 1.01 & $0.994-1.027$ & 0.219 & & & \\
\hline Gender, male/female & 0.802 & $0.530-1.212$ & 0.295 & & & \\
\hline Pathological T-category (T1-T2/T3-T4) & 4.34 & $1.376-13.688$ & $0.012^{\mathrm{b}}$ & 1.832 & $0.532-6.305$ & 0.337 \\
\hline AJCC stage (I-II/III-IV) & 3.89 & $2.127-7.118$ & $0.0^{\mathrm{b}}$ & 3.091 & $1.581-6.042$ & $0.001^{\mathrm{b}}$ \\
\hline $\begin{array}{l}\text { Tumor differentiation (moderately well } \\
\text { differentiated/poorly differentiated) }\end{array}$ & 0.721 & $0.458-1.136$ & 0.158 & & & \\
\hline Tumor size $(<5 / \geq 5 \mathrm{~cm})$ & 1.152 & $0.790-1.680$ & 0.462 & & & \\
\hline Tumor location (upper/middle/low) & 0.965 & $0.769-1.212$ & 0.761 & & & \\
\hline No. 12 a metastasis status & 1.90 & $1.221-2.956$ & $0.004^{\mathrm{b}}$ & 1.458 & $0.929-2.288$ & 0.101 \\
\hline Intravascular cancer emboli & 1.497 & $1.021-2.195$ & $0.039^{\mathrm{b}}$ & 1.137 & $0.766-1.690$ & 0.524 \\
\hline Nerve invasion & 1.417 & $0.961-2.089$ & 0.079 & 0.933 & $0.620-1.402$ & 0.738 \\
\hline
\end{tabular}

${ }^{a}$ With the use of Cox proportional hazards regression models. ${ }^{\mathrm{b}}$ Statistically significant result $(\mathrm{P}<0.05)$. AJCC, American Joint Committee on Cancer; OR, odds ratio; CI, confidence interval.

Table IV. Univariate logistic regression analysis of No. 12a lymph node metastasis.

\begin{tabular}{lccc}
\hline Factor & OR & $95 \%$ CI & P-value \\
\hline Age & 1.034 & $0.998-1.070$ & 0.064 \\
Gender & 0.797 & $0.345-1.839$ & 0.595 \\
Histological grade $\left(\mathrm{H}+\mathrm{M}^{\mathrm{a}} / \mathrm{P}+\mathrm{O}^{\mathrm{b}}\right)$ & 0.694 & $0.278-1.733$ & 0.434 \\
Tumor location (upper/middle/low) & 0.161 & $4.173-5.535$ & $<0.001^{\mathrm{c}}$ \\
AJCC stage (I+II/III+IV) & 11.96 & $1.577-90.686$ & $0.016^{\mathrm{c}}$ \\
T stage (T1+T2/T3+T4) & - & - & 0.999 \\
Intravascular cancer emboli & 2.512 & $1.112-5.676$ & $0.027^{\mathrm{c}}$ \\
Nerve invasion & 2.462 & $1.135-5.342$ & $0.023^{\mathrm{c}}$ \\
Tumor size & 0.969 & $0.453-2.075$ & 0.936 \\
\hline
\end{tabular}

${ }^{a}$ High differentiation and moderate differentiation; ${ }^{\mathrm{b}}$ poor and other types of differentiation, including mucinous and signet ring cell carcinoma; ${ }^{\mathrm{c}}$ statistically significant result $(\mathrm{P}<0.05)$. AJCC, American Joint Committee on Cancer; OR, odds ratio; CI, confidence interval.

to our clinical experiences and practices. The clinical importance and survival outcomes of patients with gastric cancer with No. 12a lymph node metastasis following D2 lymphadenectomy were also evaluated.

The data in the present study revealed that No. 12a lymph node metastasis occurred in 20\% (34/169) of the patients. This study also revealed that No. 12a lymph node metastasis was correlated with the histological grade, AJCC stage, $\mathrm{T}$ stage, lymph node metastasis, intravascular cancer emboli, nerve invasion and tumor size of the patients with gastric cancer. These results were in agreement with those of Shirong et al (14). In the present study, the rate of metastasis for the No. 12a lymph nodes was higher, predominantly since the patients in our study were diagnosed with advanced-stage disease. The percentage of stage III and IV patients with gastric cancer was $81.67 \%$ (138/169).

The present study demonstrated that survival outcomes were different between cases of No. 12a lymph node metas- tasis and those of lymph node involvement in the 7th edition AJCC-defined D2 lymphadenectomy region. Furthermore, the survival outcome was poorer in patients with No. 12a lymph node metastasis compared with those of No. 12a lymph node metastasis in stage III. However, in stage IV patients with gastric cancer, survival outcomes were similar between cases of No. 12a lymph node metastasis and those of distant metastasis. In the current study, No. 12a lymph node metastasis was linked with poor malignant tumor behavior and an advanced tumor stage. Therefore, the present results support the hypothesis that No. 12a group lymph node metastasis should be considered as distant lymph node metastasis, and this concurs with the perspective of the 7th edition AJCC regarding No. 12a lymph node metastasis. The results of the present study on No. 12a lymph node metastasis contradict those of Shirong et al (14), who proposed that No. 12a lymph node metastasis should be considered as regional lymph node metastasis. 
In the present study, Cox regression analysis demonstrated showed that the AJCC stage was independently associated with an unfavorable cumulative survival rate. Logistic regression analysis revealed that tumor location, the AJCC stage, intravascular cancer emboli and nerve invasion were associated with No. 12a lymph node metastasis.

However, the limitations of the present study include its retrospective design. The number of patients in this study was lower in comparison with those in other studies, since the scope of lymphadenectomy was strict and normative according to the scope definition of No. 12a lymph nodes. The scientificity and rationality of our hypothesis regarding No. 12a lymph nodes require further supporting evidence to substantiate them, and more randomized controlled trial studies will be required in the future.

In conclusion, the present study has demonstrated, to the best of our knowledge for the first time, a clear and practical scope definition of No. 12a group lymph nodes of gastric cancer, according to our clinical experiences and practices (Table I and Fig. 1). The survival outcome of patients with gastric cancer and No. 12a lymph node metastasis was poorer compared with that of patients with No. 12a lymph node metastasis. The results were similar in stage III patients with gastric cancer. However, the survival outcome of patients was similar with or without No. 12a lymph node metastasis in stage IV gastric cancer. Therefore, the present data suggest that No. 12a lymph node metastasis is associated with distant metastasis, and they are supportive of the 7th edition AJCC gastric cancer guidelines, which have correctly classified No. 12a lymph node metastasis as distant metastasis.

\section{Acknowledgements}

The present study was supported by the Key Project of Science and Technology Research Program in Fujian Province (no. 2012B002), the Fujian Provincial Natural Science Foundation (no. 2014J01309), the Backbone Teacher Project of Fujian Medical University (no. JGG200716), a China Non Intervention Gastric Cancer Registration Survey Clinical Research Project (no. QT-201403), a Ministry of Health Medicine Science and Technology Development and Research grant (no. W2013FZ08) and the National Clinical Key Specialty Construction Project (General Surgery) of China.

\section{References}

1. Ferlay J, Soerjomataram I, Ervik M, Dikshit R, Eser S, Mathers C, Rebelo M, Parkin DM, Forman D and Bray F: GLOBOCAN 2012v1.0, Cancer incidence and mortality worldwide: IARC CancerBase no. 11. Lyon, 2013. http://globocan.iarc.fr. Accessed April 25, 2014

2. Carneiro F: Stomach Cancer. Steward B, Wild CP (eds.) WorldCancer Report, IARC, Lyon, pp383-391, 2014.

3. Aurello P, D'Angelo F, Nigri G, Bellagamba R, Cicchini C, Ruzzetti R and Ramacciato G: Comparison between site $\mathrm{N}$-category and number $\mathrm{N}$-category for nodal staging in carcinoma of the gastroesophageal junction: Our experience and literature review. Am Surg 72: 118-123, 2006.

4. Sayegh ME, Sano T, Dexter S, Katai H, Fukagawa T and Sasako M: TNM and Japanese staging systems for gastric cancer: How do they coexist? Gastric Cancer 7: 140-148, 2004.

5. Sobin LH and Wittekind C (eds.): UICC: TNM classification of malignant tumours. 7th edition. Wiley-Liss, New York, NY, 2010.

6. Edge SBBD, Compton CC, Fritz AG, Greene FL and Trotti A: AJCC Cancer Staging Manual. 7th edition. Springer, New York, NY, 2010.

7. Degiuli M, Sasako M, Ponti A, Vendrame A, Tomatis M, Mazza C, Borasi A, Capussotti L, Fronda G and Morino M; Italian Gastric Cancer Study Group: Randomized clinical trial comparing survival after D1 or D2 gastrectomy for gastric cancer. Br J Surg 101: 23-31, 2014.

8. Jiang L, Yang KH, Guan QL, Zhao P, Chen Y and Tian JH: Survival and recurrence free benefits with different lymphadenectomy for resectable gastric cancer: A meta-analysis. J Surg Oncol 107: 807-814, 2013.

9. Japanese Gastric Cancer and Association: Japanese classification of gastric carcinoma: 3rd English edition. Gastric Cancer 14: 101-112, 2011.

10. Bilimoria KY, Bentrem DJ, Ko CY, Ritchey J, Stewart AK, Winchester DP and Talamonti MS: Validation of the 6th edition AJCC pancreatic cancer staging system: Report from the national cancer database. Cancer 110: 738-744, 2007.

11. Japanese, Biliary, Tract, Cancers and Classification: Japanese classification of Biliary tract carcinoma. English edition Biliary Tract Cancer, 2015.

12. Furukawa $H$, Imamura $H$ and Kodera $Y$ : The role of surgery in the current treatment of gastric carcinoma. Gastric Cancer 5 (Suppl 1): S13-S16, 2002.

13. Tsubono Y and Hisamichi S: Screening for gastric cancer in Japan. Gastric Cancer 3: 9-18, 2000.

14. Shirong C, Jianhui C, Chuangqi C, Kaiming W, Xinhua Z, Wu S and Yulong H: Survival of proper hepatic artery lymph node metastasis in patients with gastric cancer: Implications for D2 lymphadenectomy. PloS One 10: e0118953, 2015. 